\title{
The promoter methylation and expression of the O6-methylguanine-DNA methyltransferase gene in uterine sarcoma and carcinosarcoma
}

\author{
MATEUSZ BUJKO $^{1 *}$, MAGDALENA KOWALEWSKA $^{1 *}$, ANNA DANSKA-BIDZINSKA $^{2}$, \\ ELWIRA BAKULA-ZALEWSKA ${ }^{3}$, JANUSZ A. SIEDECKI ${ }^{1}$ and MARIUSZ BIDZINSKI ${ }^{2}$ \\ Departments of ${ }^{1}$ Molecular Biology, ${ }^{2}$ Gynecological Oncology and ${ }^{3}$ Pathology, \\ Maria Sklodowska-Curie Memorial Cancer Centre and Institute of Oncology, 02-781 Warsaw, Poland
}

Received March 5, 2012; Accepted June 8, 2012

DOI: $10.3892 / \mathrm{ol} .2012 .771$

\begin{abstract}
O6-methylguanine-DNA methyltransferase $(M G M T)$ gene promoter hypermethylation is observed in a number of solid tumors and is correlated with the silencing of $M G M T$ expression. In glioblastoma patients treated with the alkylating agent temozolomide, $M G M T$ gene methylation status was shown to have predictive value in terms of prolonged overall survival. Recently, temozolomide has demonstrated promising activity in the treatment of soft tissue sarcomas, including those of the uterus. The tissue specimens involving tumor samples and normal uterine fragments were obtained from nine patients with smooth muscle uterine sarcoma, 11 with stromal uterine sarcoma and 17 with mixed uterine tumors. MGMT gene promoter methylation was analyzed by combined bisulfite restriction analysis (COBRA) while its expression levels were assessed using the real-time reverse transcription polymerase chain reaction (qRT-PCR). MGMT promoter methylation was observed in $27 \%$ of all tumor samples analyzed. When stratified by the disease type, $55.5 \%$ (5/9) of smooth muscle sarcomas, $23.5 \%$ (4/17) of mixed uterine tumor tissues and $9 \%(1 / 11)$ of stromal sarcomas showed MGMT methylation. The $M G M T$ promoter methylation was associated with lower levels of gene expression in tumors when compared with those with an unmethylated promoter $(\mathrm{P}=0.0232)$ or normal tissues $(\mathrm{P}=0.0141)$. To conclude, $M G M T$ promoter methylation and downregulation of gene expression is observed in a fraction of carcinosarcomas and non-epithelial malignant tumors of corpus uteri. The assessment of MGMT promoter methyla-
\end{abstract}

Correspondence to: Dr Magdalena Kowalewska, Department of Molecular Biology, Maria Sklodowska-Curie Memorial Cancer Centre and Institute of Oncology, W.K. Roentgena 5, 02-781 Warsaw, Poland

E-mail: magdak@coi.waw.pl

*Contributed equally

Key words: uterine sarcoma, carcinosarcoma, O6-methylguanine-DNA methyltransferase, gene expression, gene methylation tion status may potentially identify patients who would benefit from temozolomide treatment.

\section{Introduction}

Uterine sarcomas are rare tumors, accounting for 3 to $8 \%$ of neoplasms of the uterine corpus and $1 \%$ of all tumors of the female genital tract (1). Due to the low incidence of these tumors their molecular biology, including the role of epigenetic events, is poorly understood. Uterine sarcomas are classified into smooth muscle sarcomas, stromal sarcomas and mixed uterine tumors, i.e. carcinosarcomas. Although the latter histological type is reclassified as a dedifferentiated or metaplastic form of endometrial carcinoma, it is still included in most of the studies on uterine sarcomas (2). The standard treatment of the uterine sarcomas comprises surgery and chemotherapy. Uterine sarcomas are among the most lethal uterine malignancies with poorer prognosis compared with other gynecological malignancies; 5-year survival rates remain below $50 \%$ for early stages and do not exceed $30 \%$ in the remaining stages $(3,4)$.

Recently, several adjuvant chemotherapy regimens have been reported in the treatment of soft tissue sarcomas, including sarcomas of the uterus. Some of the trials were based on the alkylating agents, including carmustine and temozolomide (TMZ). The regiment combining carmustine with $\mathrm{O}^{6}$-benzylguanine did not result in objective treatment response in 12 enrolled patients with soft tissue sarcoma (5). The initial results concerning TMZ-based therapy as a second-line treatment in 31 patients with advanced soft tissue sarcoma did not show activity of the drug (6). The trial involving patients with soft tissue sarcomas not subjected to standard chemotherapy revealed only minimal efficacy of TMZ (7). Another trial on TMZ demonstrated a modest activity against previously treated unresectable or metastatic soft tissue sarcomas (8). Notably, all responding patients had leiomyosarcoma (of uterine or non-uterine origin) (8).

Better results were obtained in 2005 by the Spanish Group for Research on Sarcomas with the prolonged course of TMZ which had activity in patients with pretreated soft tissue sarcomas (9). Notably, a response was observed in 5 of 11 patients who had gynecological leiomyosarcoma and in one 
of two patients with mixed mullerian tumors. The results of the study on the uterine leiomyosarcoma were published in the same year, revealing therapeutic benefit of TMZ in patients with metastatic unresectable disease (10). Of 19 patients pretreated with doxorubicin who underwent TMZ-based therapy, two patients achieved almost complete response and eight showed stabilization of the disease. In a recent study of Ferriss et al (11) a clinical benefit of TMZ was achieved in five out of six patients with advanced and recurrent uterine leiomyosarcoma. This therapeutic benefit was associated with silencing of the O6-methylguanine-DNA methyltransferase (MGMT) expression as determined by immunohistochemistry. All the above mentioned studies revealed good tolerance to TMZ (6-11).

The MGMT gene has been shown to be epigenetically downregulated in several solid tumors. Aberrant promoter hypermethylation of the $M G M T$ has been associated with the lack of its mRNA expression, the loss of MGMT protein (12) and loss of enzymatic activity (13). MGMT encodes DNA repair protein, an enzyme responsible for the direct removal of alkylating adducts from guanines. The silencing of the gene contributes to the reduction of the genome stability and sensitizes tumor cells to alkylating agents, including dacarbazine, carmustine and TMZ. The main therapeutic target of these drugs are the nitrogen bases of DNA and the most important cytotoxic derivate of their action on the nitrogen bases is O6-methyl-guanine. Alkylation of the guanine leads to an accumulation of the replication errors during the S-phase of the cell cycle and, as a consequence, to the cell cycle arrest and/or apoptosis. The high degree of removal of alkyl adducts causes the resistance to treatment.

As has been shown in tumor cell line-based studies, MGMT prevents TMZ-induced cell death by removing alkyl adducts from the $\mathrm{O}^{6}$ position of guanine (14). Thus, tumor cells expressing MGMT are resistant to alkylating agents, while those that lack the enzyme appear to be chemosensitive. The predictive value of $M G M T$ epigenetic silencing is well documented for glioblastoma treatment with TMZ. The methylation of the promoter of the gene was shown to correlate with improved prognosis in several independent trials and is being considered as a potential stratification marker of the response to TMZ-based therapy (15). However, to date no formal recommendation has been proposed as to the use of this marker in the clinical setting. In melanomas treated with TMZ, the MGMT methylation was associated with improved tolerance to treatment, however not with survival (16).

This study aimed to evaluate the frequency of $M G M T$ promoter methylation, as well as to assess its possible correlation with the expression levels of the gene, in carcinosarcomas and non-epithelial malignant tumors of corpus uteri.

\section{Materials and methods}

Patients. A total of nine patients treated for smooth muscle uterine sarcoma, 11 for stromal uterine sarcoma and 17 for mixed uterine tumors in the Maria Sklodowska-Curie Memorial Cancer Centre and Institute of Oncology in Warsaw between January 2009 and December 2010 were enrolled in the present study. The selected patients' characteristics are presented in Table I. The study was approved by the Independent
Ethics Committee of the Maria Sklodowska-Curie Memorial Cancer Centre and Institute of Oncology in Warsaw and all patients provided informed consent. Tissue specimens were divided into two parts: one part was examined histologically, the other was frozen in liquid nitrogen and stored at $-70^{\circ} \mathrm{C}$ until nucleic acid isolation. In addition to 37 tumor tissue samples, 19 samples of normal uterine tissue were also obtained from patients enrolled in the study.

DNA methylation analysis. MGMT promoter methylation analysis was performed using the combined bisulfite restriction analysis (COBRA).

DNA was isolated from $\sim 50 \mathrm{mg}$ of pulverized (with the Microdismembrator II, B Braun Biotech International, Melsungen, Germany) tumor samples using NucloSpin Tissue kit (Macherey-Nagel, Düren, Germany), according to the manufacturer's instructions. DNA quantity was measured using NanoDrop 2000 (ThermoScientific, Waltham, MA, USA). DNA ( $1 \mu \mathrm{g})$ was bisulfite converted using EpiTect kit (Qiagen, Hilden, Germany) according to the manufacturer's instructions. Converted DNA was eluted with $40 \mu \mathrm{l}$ of water. MGMT promoter region [chr10:131155461-131155570 genome location as determined by UCSC Genome Browser Database (http://genome.ucsc.edu/) Human March 2006 (hg18) assembly] region was amplified using previously reported PCR primers (17). The reaction volume of $15 \mu \mathrm{l}$ contained 1X PCR buffer, $2 \mathrm{mM} \mathrm{MgCl}$, $0.25 \mathrm{mM}$ dNTPs, $0.2 \mathrm{mM}$ each primer, $0.5 \mathrm{U}$ of FastStart DNA Polymerase (Roche Applied Science, Mannheim, Germany) and $1 \mu \mathrm{l}$ of bisulfite-treated DNA as a template. The cycling conditions were as follows: initial denaturation at $94^{\circ} \mathrm{C}$ for $3 \mathrm{~min}$; followed by 38 cycles of $30 \mathrm{sec}$ at $94^{\circ} \mathrm{C}, 40 \mathrm{sec}$ annealing at $58^{\circ} \mathrm{C}$ and $50 \mathrm{sec}$ at $72^{\circ} \mathrm{C}$; then final elongation for $7 \mathrm{~min}$ at $72^{\circ} \mathrm{C}$. Subsequently, $8 \mu \mathrm{l}$ of PCR products were digested overnight with HpyCH4IV (TaiI) restriction enzyme (New England Biolabs, Ipswich, MA, USA) which cleaves sequences containing CpG dinucleotides. Restriction fragments were electrophoresed in $10 \%$ polyacrylamide gel (acryl/bis 19:1) and visualized with ethidium bromide. The presence of 61- and 39-bp DNA fragments on the gel (the shortest 15-bp DNA fragment was not visible in certain samples due to the low band intensity) indicates the occurrence of methylated MGMT variant. The unmethylated DNA variants, as wells as native DNA (not subjected to bisulfite conversion), have no restriction sites for the chosen enzyme in the analyzed region which excludes the occurrence of false positive results.

DNA isolated from the blood sample of a healthy donor was methylated in vitro with SssI DNA methyltransferase (New England Biolabs) and used as a positive (methylated) control. The same DNA sample after whole genome amplification (GenomiPhi, GE Healthcare, Piscataway, NJ, USA) was used as a negative (unmethylated) control.

Expression analysis. MGMT mRNA level was evaluated using the real-time reverse transcription polymerase chain reaction (qRT-PCR).

Total RNA from $\sim 50 \mathrm{mg}$ of pulverized (with the Microdismembrator II, B Braun Biotech International) tumor and normal uterine samples was extracted using RNeasy Mini kit with on-column DNase digestion (Qiagen) according to 


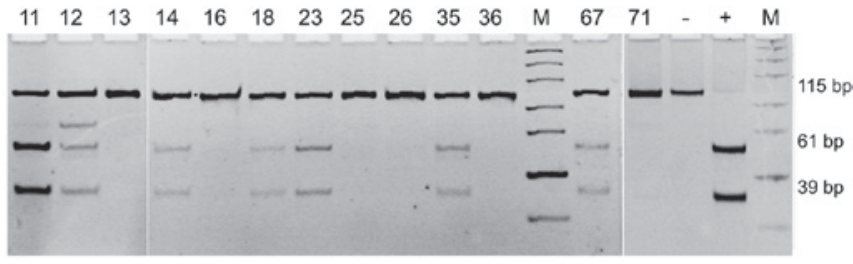

Figure 1. The representative results of $M G M T$ promoter methylation analysis. Lanes 12-71, tumor samples; -, negative control (whole genome amplified DNA from the blood sample of a healthy donor); +, positive control (in vitro methylated DNA from the blood sample of a healthy donor); M, molecular weight marker; MGMT, O6-methylguanine-DNA methyltransferase.

the manufacturer's instructions. RNA quantity was measured using NanoDrop (ThermoScientific), while the overall RNA quality was assessed by electrophoresis on a denaturing agarose gel (FlashGel, Lonza, Rockland, ME, USA). The RNA samples ( $1 \mu \mathrm{g}$ each) were reverse-transcribed using the $\mathrm{RT}^{2}$ First Strand kit (SA Biosciences, Hilden, Germany) according to the manufacturer's instructions. Quantitative real-time PCR was performed in triplets using ABI Prism 7000 Sequence Detection System (Applied Biosystems, Carlsbad, CA, USA). The reaction mixture of $25 \mu \mathrm{l}$ contained $2.5 \mu \mathrm{l}$ of $15 \mathrm{X}$ diluted cDNA template, 1X Power SYBR Green PCR Master Mix (Applied Biosystems) and $0.1 \mathrm{mM}$ of the forward and reverse primers. PCR was performed as follows: precycling hold at $95^{\circ} \mathrm{C}$ for $10 \mathrm{~min}, 45$ cycles: $95^{\circ} \mathrm{C}$ for $30 \mathrm{sec}$ and $60^{\circ} \mathrm{C}$ for $60 \mathrm{sec}$. To assess the reaction specificity, the amplification products were subjected to melting curve analysis.

$U B C$ was used as a reference gene, as its stable expression in carcinosarcoma tumors and non-epithelial malignant tumors of the corpus uteri as well as in normal uterine tissues has been recently demonstrated (18). Primer sequences for the $M G M T$ and $U B C$ were obtained from the qPimerDepot database (19). These were: $M G M T$ forward, CTCCGGACCTCCGAGAAC, and $M G M T$ reverse, GTCTGCACGAAATAAAGC, producing 94-bp amplicons; as well as $U B C$ forward, TTGCCTTGACATTCTCGATG, and $U B C$ reverse, ATCGCTGTGATCGTCACTTG, producing 108-bp amplicons.

Raw data were analyzed using ABI Prism 7000 SDS Software Version 1.1 (Applied Biosystems). Relative expression levels were calculated using the $2^{-\Delta \mathrm{Ct}}$ method, where $\Delta \mathrm{Ct}$ was defined as a difference between $\mathrm{Ct}$ value for $M G M T$ and $U B C$ reference gene.

Statistical analysis. The Chi-square test was used to compare MGMT methylation frequencies among the three histopathological subtypes of the analyzed tumors. The difference in the $M G M T$ expression levels between $M G M T$-methylated and unmethylated tumors and normal uterine tissues was assessed with the use of a two-sided Mann-Whitney U test with a significance threshold level $\alpha=0.05$. The values of the $M G M T$ expression levels were visualized in a plot using GraphPadPrism (La Jolla, CA, USA).

\section{Results}

MGMT promoter methylation was observed in $27 \%$ (10/37) of tumors obtained from all the patients enrolled into our study.

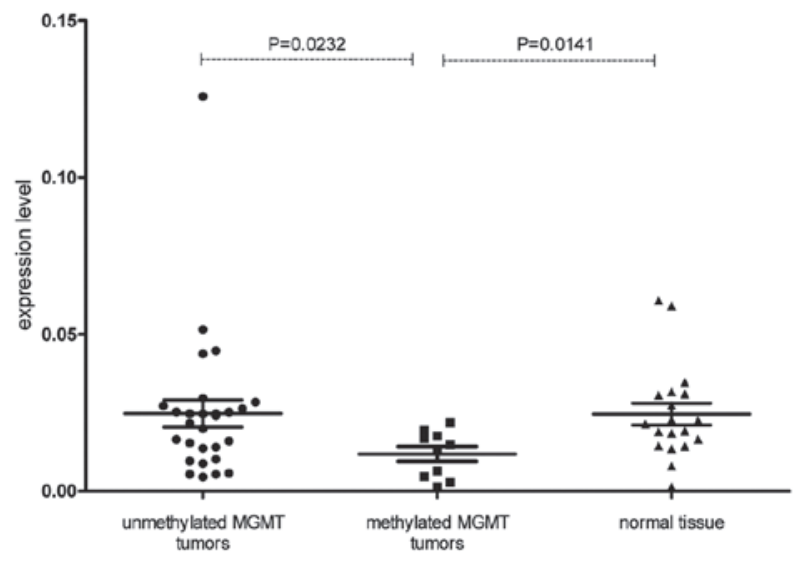

Figure 2. The MGMT expression levels in tumors with methylated or unmethylated MGMT and normal uterine tissues; MGMT, O6-methylguanine-DNA methyltransferase. Horizontal lines indicate standard error of the mean (SEM).

In three cases that showed MGMT promoter methylation, an additional band on the gel was observed indicating incomplete digestion and therefore incomplete promoter methylation (Fig. 1, sample 12). When stratified by the disease type, $55.5 \%$ $(5 / 9)$ of smooth muscle sarcomas, $23.5 \%$ (4/17) of mixed uterine tumor tissues and 9\% (1/11) of stromal sarcomas showed MGMT methylation. The difference in frequency of $M G M T$ promoter methylation in smooth muscle sarcomas compared with the two other subtypes of the analyzed tumors was statistically significant $(\mathrm{P}=0.0489)$.

The $M G M T$ expression level was significantly lower in tumor samples with gene promoter methylation when compared with unmethylated tumor tissues $(\mathrm{P}=0.0232)$. The $M G M T$ expression level was also significantly lower in tumor samples with gene promoter methylation than in the normal uterine samples obtained from the same patients $(\mathrm{P}=0.0141$; Fig. 2). The promoter methylation status and values of $M G M T$ expression levels for individual uterine sarcoma and carcinosarcoma tumors are provided in Table I.

No difference between $M G M T$-unmethylated tumors and normal samples was observed.

\section{Discussion}

MGMT promoter methylation status has previously been shown to correlate with the downregulation of the gene expression in different types of solid tumors (20). The aim of this study was to evaluate the frequency of $M G M T$ promoter methylation and its correlation with the gene expression status in carcinosarcomas and non-epithelial malignant tumors of the corpus uteri. We observed the methylated MGMT variant in a relatively high fraction of tumors $(27 \%)$, being the highest in smooth muscle sarcomas where the gene was methylated in over half of the cases. The tumors with $M G M T$ promoter methylation showed a significantly lower gene expression level than tumors with an unmethylated promoter as well as normal uterine tissue samples.

A number of cell line-based experiments have revealed the inverse correlation between the MGMT expression and the cytotoxic effect of TMZ. These observations have been 
Table I. Individual patient data.

\begin{tabular}{|c|c|c|c|c|c|}
\hline Patient & $\begin{array}{c}\text { Age } \\
\text { (years) }\end{array}$ & $\begin{array}{c}M G M T \text { methylation } \\
\text { status }\end{array}$ & $\begin{array}{l}M G M T \text { expression } \\
\text { level }\end{array}$ & $\begin{array}{l}\text { Tumor } \\
\text { type }\end{array}$ & $\begin{array}{l}\text { Tumor histology/ } \\
\text { histological grade }\end{array}$ \\
\hline \multicolumn{6}{|c|}{ Mixed uterine tumors } \\
\hline 3 & 75.5 & Negative & 0.010 & Recurrent & Carcinosarcoma heterologous ${ }^{\mathrm{a}}$ \\
\hline 4 & 54.1 & Negative & 0.025 & Primary & Carcinosarcoma heterologous ${ }^{\mathrm{b}}$ \\
\hline 5 & 79.5 & Negative & 0.009 & Primary & Carcinosarcoma heterologous \\
\hline 11 & 61.4 & Positive & 0.017 & Primary & Carcinosarcoma homologous \\
\hline 14 & 23.2 & Positive & 0.015 & Primary & Adenosarcoma homologous \\
\hline 18 & 66.4 & Positive & 0.013 & Primary & Carcinosarcoma heterologous \\
\hline 24 & 66.6 & Negative & 0.025 & Primary & Carcinosarcoma heterologous \\
\hline 25 & 61.0 & Negative & 0.020 & Recurrent & Carcinosarcoma homologous \\
\hline 30 & 64.3 & Negative & 0.025 & Primary & Carcinosarcoma heterologous \\
\hline 33 & 61.6 & Negative & 0.022 & Recurrent & Carcinosarcoma heterologous \\
\hline 39 & 54.7 & Negative & 0.044 & Primary & Adenosarcoma homologous \\
\hline 44 & 68.0 & Negative & 0.030 & Primary & Adenosarcoma heterologous \\
\hline 47 & 65.7 & Negative & 0.014 & Primary & Carcinosarcoma homologous \\
\hline 51 & 56.4 & Negative & 0.005 & Primary & $\begin{array}{l}\text { Mixed endometrial stromal and } \\
\text { smooth muscle tumor }\end{array}$ \\
\hline 66 & 55.1 & Negative & 0.045 & Primary & Carcinosarcoma homologous \\
\hline 67 & 59.7 & Positive & 0.003 & Primary & Adenosarcoma homologous \\
\hline 71 & 55.5 & Negative & 0.126 & Recurrent & Adenosarcoma (dediff) \\
\hline \multicolumn{6}{|c|}{ Smooth muscle sarcomas } \\
\hline 1 & 36.6 & Positive & 0.022 & Primary & Rhabdomyosarcoma \\
\hline 2 & 52.6 & Positive & 0.001 & Recurrent & Leiomyosarcoma/G3 \\
\hline 12 & 56.3 & Positive & 0.005 & Primary & Leiomyosarcoma/G3 \\
\hline 23 & 63.2 & Positive & 0.006 & Recurrent & Leiomyosarcoma/G2 \\
\hline 26 & 45.8 & Negative & 0.025 & Recurrent & Leiomyosarcoma/G3 \\
\hline 28 & 51.5 & Negative & 0.005 & Recurrent & Leiomyosarcoma/G2 \\
\hline 35 & 57.5 & positive & 0.018 & Recurrent & Leiomyosarcoma/G2 \\
\hline 37 & 25.5 & Negative & 0.016 & Recurrent & STUMP \\
\hline 63 & 40.5 & Negative & 0.005 & Recurrent & Leiomyosarcoma/G3 \\
\hline \multicolumn{6}{|c|}{ Stromal uterine sarcomas } \\
\hline 6 & 76.7 & Negative & 0.024 & Recurrent & Endometrial stromal sarcoma, low grade \\
\hline 8 & 59.5 & Positive & 0.019 & Primary & Undifferentiated endometrial sarcoma \\
\hline 13 & 60.1 & Negative & 0.025 & Recurrent & Undifferentiated endometrial sarcoma \\
\hline 16 & 43.3 & Negative & 0.017 & Recurrent & Endometrial stromal sarcoma, low grade \\
\hline 17 & 74.8 & Negative & 0.006 & Primary & Undifferentiated endometrial sarcoma \\
\hline 31 & 51.0 & Negative & 0.014 & Primary & Sarcoma stromale, low grade \\
\hline 36 & 64.6 & Negative & 0.052 & Primary & Undifferentiated endometrial sarcoma \\
\hline 38 & 44.8 & Negative & 0.010 & Primary & Endometrial stromal sarcoma low grade \\
\hline 52 & 78.1 & Negative & 0.027 & Primary & Undifferentiated endometrial sarcoma \\
\hline 62 & 53.4 & Negative & 0.026 & Primary & Undifferentiated endometrial sarcoma \\
\hline 64 & 68.9 & Negative & 0.028 & Primary & Undifferentiated endometrial sarcoma \\
\hline
\end{tabular}

aheterologous tumor, representing the malignant counterparts that normally do not occur in the uterus; bhomologous tumor, representing the malignant counterparts of tissues indigenous to the uterus. MGMT, O6-methylguanine-DNA methyltransferase; STUMP, smooth muscle tumor of uncertain malignant potential.

confirmed in clinical trials. In the study by Ferriss et al (11) on the effectiveness of TMZ in uterine leiomyosarcomas treatment, the MGMT expression was inversely correlated with treatment response.
Using the MGMT promoter methylation status as a predictive biomarker has certain advantages. As cytosine methylation is a stable covalent modification, it may be analyzed in a wide range of tissue samples, including formalin-fixed and 
paraffin-embedded (FFPE) tissues. FFPE samples are probably the most accessible clinical tissue material for molecular analysis, although inappropriate for the mRNA expression analysis. Currently available laboratory techniques allow relatively fast and sensitive $M G M T$ methylation detection with qualitative or quantitative results. Compared with immunohistochemical expression analysis, the determination of MGMT methylation status is not dependent on subjective microscopic evaluation and if a quantitative technique is applied, more precise quantitative results may be achieved.

TMZ-based therapy is the current standard in the treatment of glioblastoma patients and MGMT methylation status has already been shown to be strong predictive factor of significantly longer progression free survival and overall survival of patients with methylation of the gene promoter (21). The systematic comparison of the application of immunohistochemical staining with the promoter methylation analysis in the glioblastoma patients treated with TMZ revealed the superiority of methylation analysis as a survival predictive factor (22).

As the group of patients enrolled in our study is relatively small, the findings have value as preliminary results. However, the presence of $M G M T$ promoter methylation in a notable proportion of patients and the observation that gene methylation is associated with the downregulation of the gene expression levels indicate that methylation analysis should be included in the clinical trials on the effectiveness of TMZ in patients with uterine sarcoma and carcinosarcoma. The results of the present study advocate the use of TMZ in uterine leiomyosarcoma treatment and also suggest that a smaller percentage of patients with stromal sarcoma and carcinosarcoma may benefit from this type of therapy. The qualitative techniques for MGMT promoter methylation detection, including COBRA that was used in our study, potentially allow prediction of the patients' response to TMZ-based treatment and thus their stratification.

To conclude, as TMZ-based chemotherapy showed promising results in recently reported trials on the treatment of soft tissue sarcomas, determination of $M G M T$ promoter methylation status may have significant clinical implications in a fraction of patients with carcinosarcoma and non-epithelial malignant tumors of the corpus uteri. Such studies should be applied in the clinical practice and ultimately contribute to future therapeutic strategies for these rare gynecological tumors.

\section{Acknowledgements}

This study was supported by the research grant No. NN 407 125937 from the Ministry of Science and Higher Education.

\section{References}

1. Brooks SE, Zhan M, Cote T and Baquet CR: Surveillance, epidemiology, and end results analysis of 2677 cases of uterine sarcoma 1989-1999. Gynecol Oncol 93: 204-208, 2004.

2. D'Angelo E and Prat J: Uterine sarcomas: a review. Gynecol Oncol 116: 131-139, 2010.
3. Major FJ, Blessing JA, Silverberg SG, et al: Prognostic factors in early-stage uterine sarcoma. A Gynecologic Oncology Group study. Cancer 71 (Suppl 4): 1702-1709, 1993.

4. Zagouri F, Dimopoulos AM, Fotiou S, Kouloulias V and Papadimitriou CA: Treatment of early uterine sarcomas: disentangling adjuvant modalities. World J Surg Oncol 7: 38, 2009.

5. Ryan CW, Dolan ME, Brockstein BB, et al: A phase II trial of O6-benzylguanine and carmustine in patients with advanced soft tissue sarcoma. Cancer Chemother Pharmacol 58: 634-639, 2006.

6. Woll PJ, Judson I, Lee SM, et al: Temozolomide in adult patients with advanced soft tissue sarcoma: a phase II study of the EORTC Soft Tissue and Bone Sarcoma Group. Eur J Cancer 35: 410-412, 1999.

7. Trent JC, Beach J, Burgess MA, et al: A two-arm phase II study of temozolomide in patients with advanced gastrointestinal stromal tumors and other soft tissue sarcomas. Cancer 98: 2693-2699, 2003.

8. Talbot SM, Keohan ML, Hesdorffer M, et al: A phase II trial of temozolomide in patients with unresectable or metastatic soft tissue sarcoma. Cancer 98: 1942-1946, 2003.

9. Garcia del Muro X, Lopez-Pousa A, Martin J, et al; Spanish Group for Research on Sarcomas: A phase II trial of temozolomide as a 6-week, continuous, oral schedule in patients with advanced soft tissue sarcoma: a study by the Spanish Group for Research on Sarcomas. Cancer 104: 1706-1712, 2005.

10. Anderson S and Aghajanian C: Temozolomide in uterine leiomyosarcomas. Gynecol Oncol 98: 99-103, 2005.

11. Ferriss JS, Atkins KA, Lachance JA, Modesitt SC and Jazaeri AA: Temozolomide in advanced and recurrent uterine leiomyosarcoma and correlation with o6-methylguanine DNA methyltransferase expression: a case series. Int J Gynecol Cancer 20: $120-125,2010$.

12. Esteller M, Hamilton SR, Burger PC, Baylin SB and Herman JG: Inactivation of the DNA repair gene O6-methylguanine-DNA methyltransferase by promoter hypermethylation is a common event in primary human neoplasia. Cancer Res 59: 793-797, 1999.

13. Herfarth KK, Brent TP, Danam RP, et al: A specific CpG methylation pattern of the MGMT promoter region associated with reduced MGMT expression in primary colorectal cancers. Mol Carcinog 24: 90-98, 1999.

14. Kaina B, Fritz G, Mitra S and Coquerelle T: Transfection and expression of human O6-methylguanine-DNA methyltransferase (MGMT) cDNA in Chinese hamster cells: the role of MGMT in protection against the genotoxic effects of alkylating agents. Carcinogenesis 12: 1857-1867, 1991.

15. Franceschi E, Tosoni A, Pozzati E and Brandes AA: Association between response to primary treatments and MGMT status in glioblastoma. Expert Rev Anticancer Ther 8: 1781-1786, 2008.

16. Hassel JC, Sucker A, Edler L, et al: MGMT gene promoter methylation correlates with tolerance of temozolomide treatment in melanoma but not with clinical outcome. Br J Cancer 103: 820-826, 2010.

17. Smith E, Jones ME and Drew PA: Quantitation of DNA methylation by melt curve analysis. BMC Cancer 9: 123, 2009.

18. Kowalewska M, Danska-Bidzinska A, Bakula-Zalewska E and Bidzinski M: Identification of suitable reference genes for gene expression measurement in uterine sarcoma and carcinosarcoma tumors. Clin Biochem 45: 368-371, 2012.

19. Cui W, Taub DD and Gardner K: qPrimerDepot: a primer database for quantitative real time PCR. Nucleic Acids Res 35: D805-809, 2007.

20. Jacinto FV and Esteller M: MGMT hypermethylation: a prognostic foe, a predictive friend. DNA Repair (Amst) 6: 1155-1160, 2007.

21. Hegi ME, Diserens AC, Gorlia T, et al: MGMT gene silencing and benefit from temozolomide in glioblastoma. N Engl J Med 352: 997-1003, 2005

22. Preusser M, Charles Janzer R, Felsberg J, et al: Anti-O6methylguanine-methyltransferase (MGMT) immunohistochemistry in glioblastoma multiforme: observer variability and lack of association with patient survival impede its use as clinical biomarker. Brain Pathol 18: 520-532, 2008. 